\title{
Anelastic Behavior in Crystalline Nanowires
}

\author{
Guangming Cheng ${ }^{1}$ and Yong Zhu ${ }^{1}$ \\ 1. Department of Mechanical and Aerospace Engineering, North Carolina State University, Raleigh, \\ USA.
}

NWs exhibit a host of novel properties that are being exploited for many applications including energy harvesting and storage, flexible/stretchable electronics, sensing, and nanoelectromechanical systems. So far a vast majority of research on mechanical properties of NWs has been focused on size-dependent elastic modulus and strength, with very few studies on time-dependent responses [1]. This work reports an unexpected phenomenon of anelastic relaxation in crystalline NWs under bending [2]. Anelastic materials exhibit gradual full recovery of deformation once a load is removed, leading to efficient dissipation of internal mechanical energy. As a consequence, anelastic materials are being investigated for energy damping applications. At macroscopic scale, however, anelaticity is usually very small or negligible, especially in single-crystalline materials. Here we show that single-crystalline $\mathrm{ZnO}$ can exhibit anelastic behavior that is up to four orders of magnitude larger than the largest anelasticity observed in bulk materials, with a recovery time-scale in the order of minutes. In-situ scanning electron microscope (SEM) tests of individual NWs showed that, upon removal of the bending load and instantaneous recovery of the elastic strain, a substantial portion of the total strain gradually recovers with time (Fig. 1). We attribute the observed large anelasticity to stress-gradient-induced migration of point defects, as supported by quantitative electron energy loss spectroscopy (EELS) measurements [2] and also by the fact that no anelastic behavior could be observed under tension.

The bending tests were performed at the room temperature using a microelectromechanical system (MEMS) based nanomechanical testing stage inside an SEM [3,4]. After the NW was held at a bent configuration for certain time (holding time), the MEMS actuator was retracted and the shape of the NW was monitored in real time (Fig. 1). It can be seen that a large portion of the bending strain recovered instantaneously while the rest recovered gradually with time, shown in Fig. 1a. Fig. 1b shows the evolution of the anelastic strain of a $\mathrm{ZnO} \mathrm{NW}$ (54 nm in diameter) for an initial (total) bending strain of $1.94 \%$ with six different durations of holding time. Here the initial or total strain is the sum of anelastic strain and elastic strain, right before the load is removed. It can be seen that the recovery of the anelastic strain depends on the holding time - the shorter the holding time, the faster the recovery. Fig. 1c shows the evolution of the anelastic strain for a holding time of 15 minutes under five different initial strains. Larger initial strain led to larger anelastic strain. The anelastic strain almost fully recovered with time (with holding time of 15 minutes and recovery time up to 40 minutes). The anelastic strain was as large as $0.64 \%$ (in the case of $4.1 \%$ total strain). The observed anelasticity was attributed to a type of nonlinear Gorsky relaxation that involves the diffusion of two types of point defects under an inhomogeneous stress field. A theoretical model was developed to understand the relationship between the diffusion of point defects and the measured anelastic behavior [2]. Finite element simulations were performed to simulate the bending and relaxation processes under the same conditions as in the experiments. Two defect types with corresponding diffusivities and initial concentrations were considered. The evolution of the anelastic strain was calculated as functions of the initial strain and holding time, and compared to the experimental results (Fig. 1). Finally, to assess if the observed that anelasticity is present in other crystalline NWs with point defects, we tested p-doped Si NWs and Ag NWs and observed similar anelastic relaxation [5]. 
References:

[1] Q. Qin et al, Nature communications 6 (2015), p. 5983.

[2] G Cheng et al, Nature Nanotechnology 10 (2015), p. 687.

[3] Y Zhu and HD Espinosa, Proceedings of the National Academy of Sciences of the United States of America 102 (2005), p. 14503.

[4] G Cheng, "In Situ Investigation of Deformation Mechanisms in Crystalline Nanowires", PhD Thesis, 2016.

[5] The authors acknowledge funding from the National Science Foundation (NSF) under Award Nos.

CMMI-1030637 and 1301193, and the use of the Analytical Instrumentation Facility (AIF) at North

Carolina State University (NSF Award No. ECCS-1542015).
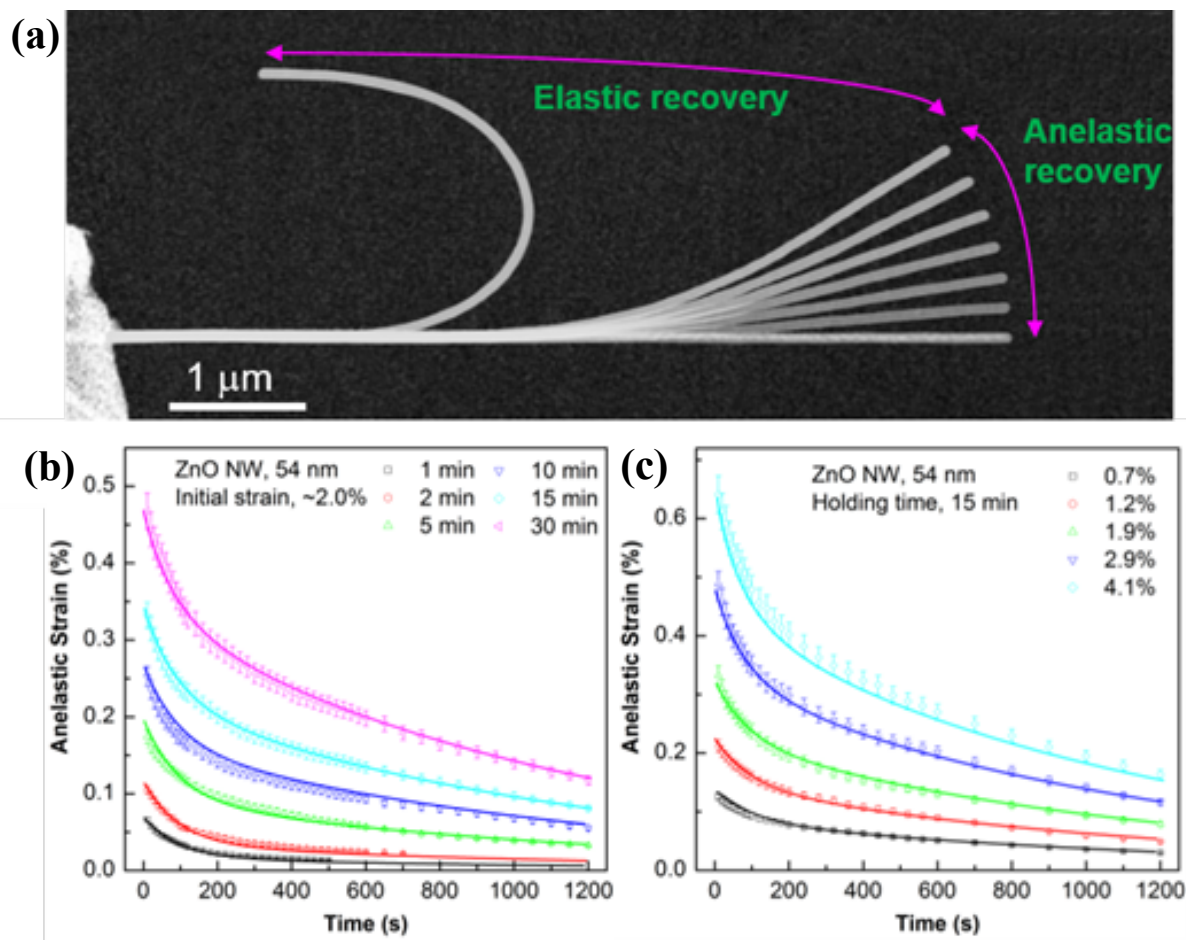

Figure 1. Recovery and damping behaviors of a $\mathrm{ZnO}$ NW. (a) series of images showing the elastic and anelastic recovery. (b) Anelastic strain as a function of recovery time for six different durations of holding time. The NW diameter was $54 \mathrm{~nm}$ and the initial bending strain was $1.94 \%$. (c) Anelastic strain as a function of recovery time for five different initial bending strains. The holding time was 15 minutes in all five cases. Solid lines in both (a) and (b) represent the fitting data from finite element analysis. Error bars for strain measurement in (b) and (c) were $\sim 5.4 \%$. 